\title{
Simplified Regional Citrate Anticoagulation Using a Calcium-Containing Replacement Solution for Continuous Renal Replacement Therapy: A Randomized Controlled Trial
}

\section{Tiantian Wei}

West China School of Medicine: Sichuan University West China Hospital

\section{Xin Tang}

West China School of Medicine: Sichuan University West China Hospital

Ling Zhang ( $\nabla$ zhanglinglzy@163.com)

West China Hospital of Sichuan University https://orcid.org/0000-0003-1155-4802

Li Lin

West China School of Medicine: Sichuan University West China Hospital

\section{Peiyun Li}

West China School of Medicine: Sichuan University West China Hospital

\section{Fang Wang}

West China School of Medicine: Sichuan University West China Hospital

\section{Ping Fu}

West China School of Medicine: Sichuan University West China Hospital

\section{Research}

Keywords: Regional citrate anticoagulation, calcium-containing replacement solution, randomized clinical trial, CRRT, hemodiafiltration

Posted Date: September 29th, 2020

DOI: https://doi.org/10.21203/rs.3.rs-82684/v1

License: (9) This work is licensed under a Creative Commons Attribution 4.0 International License. Read Full License 


\section{Abstract}

Introduction A simplified protocol for regional citrate anticoagulation (RCA) using a commercial calciumcontaining replacement solution, without continuous calcium infusion, is more efficient to be used in continuous renal replacement therapy (CRRT). We aim to design a randomized clinical trial (RCT) to compare the safety and efficacy between calcium-free and calcium-containing replacement solution in CRRT with RCA.

Methods Of the 64 patients receiving RCA-based post-dilution continuous venovenous hemodiafiltration (CVVHDF) enrolled from 2017-2019, 35 patients were randomized to calcium-containing group and 29 to calcium-free replacement solution group. Primary endpoint was circuit lifespan. Secondary endpoints included mortality, kidney function recovery and complications. The amount of $4 \%$ trisodium citrate solution infusion was recorded. Serum and effluent total and ionized calcium concentration were measured during CVVHDF.

Results Total 149 circuits (82 in calcium-containing group and 67 in calcium-free group) and 7609 circuit hours (4335 versus 3274 hours) were included. Mean circuit lifespan was 58.1 hours (95\% Cl 53.8-62.4) in calcium-containing group versus 55.3 hours $(95 \% \mathrm{Cl} 49.7-60.9$, log rank $P=0.89)$ in calcium-free group. The serum total ( $\mathrm{tCa}$ ) and ionized (iCa) calcium concentration was slightly lower in calcium-containing group during CRRT, whereas the post-filter iCa concentration was lower in calcium-free group. Moreover, mean $4 \%$ trisodium citrate solution infusion doses had no difference between groups $(171.1 \pm 15.9$ versus $169.0 \pm 15.1 \mathrm{ml} / \mathrm{h}, P=0.49)$. The mortality $(40.0 \%$ versus $44.8 \%, P=0.70)$ and kidney function recovery rates (54.3\% versus $48.3 \%, P=0.63$ ) were similar in calcium-containing and calcium-free group during hospitalization, respectively. Six ( 3 in each group) patients showed the signs of citrate accumulation in this study.

Conclusions When compared with calcium-free replacement solution, RCA based CVVHDF with calciumcontaining replacement solution had similar circuit lifespan, hospital mortality and kidney outcome. Since the calcium-containing solution obviates the need for a separate venous catheter and large dose of intravenous calcium solution preparation for continuous calcium supplement, it is more convenient to be applied in RCA-CRRT practice.

Trial registration Chinese Clinical Trial Registry. ChiCTR-IPR-17012629, registered on 10th September 2017. http://www.chictr.org.cn/showprojen.aspx?proj=17644

\section{Key Massages}

A simplified protocol for regional citrate anticoagulation (RCA) using a commercial calcium-containing replacement solution, obviates the need for large dose of intravenous calcium solution preparation for continuous calcium supplement, it is more convenient to be applied in RCA-CRRT practice. 
Compared with calcium-free replacement solution, calcium-containing replacement solution had similar circuit lifespan and patient outcome. It is safety and efficacy to be used in clinical practice.

\section{Introduction}

Continuous renal replacement therapies (CRRT) are widely applied in critically ill patients with acute kidney injury (AKI) or end stage renal disease (ESRD) [1]. Various anticoagulation strategies, such as citrate and heparin, are used to prevent extracorporeal circuit from clotting [2, 3]. Regional citrate anticoagulation (RCA), which can reduce the risk of circuit loss and bleeding, has been proven to be more effective and safer than systemic or regional heparin anticoagulation for CRRT [2, 4]. Therefore, the 2012 Kidney Disease Improving Global Outcomes (KDIGO) Clinical Practice Guidelines for Acute Kidney Injury recommended RCA as the first choice in CRRT without contraindications for citrate (2B) [5].

Citrate acts as an anticoagulation by chelating the ionized calcium in the extracorporeal circuit and then blocking the coagulation cascade [6, 7]. Since traditional RCA usually applies calcium-free solutions, persistent and large dose of systemic calcium infusion is needed to replace calcium loss in effluent fluid and prevent hypocalcemia [8-10]. However, the shortage of intravenous calcium in some countries and complex protocols of calcium-free solution as well as the increasing workload have limited the use of RCA [11-13]. In order to make RCA more convenient to be applied in CRRT in our hospital, we developed a simplified RCA-based continuous venovenous hemofiltration (CVVH) protocol which utilized $4 \%$ trisodium citrate solution and a commercial calcium-containing replacement solution [14]. Together with other previous studies, the calcium-containing solution has been confirmed to be safe and effective without a continuous calcium infusion in RCA and it has become widespread [11, 15-17].

Whereas it remains unknown whether RCA with calcium-free solution or calcium-containing solution are equivalent in RCA-based CRRT in terms of prolonging circuit life or which one of these two solutions is superior. It is unclear whether these two types of solutions have different effects on hemofilters or even the patients' clinical outcome. To address these questions, we conducted a prospective single-center randomized clinical trial (RCT) to observe the efficacy and safety of RCA using calcium-containing solution. This study may provide evidence that simplified RCA using a calcium-containing replacement solution could be a good choice for patients.

\section{Material And Methods}

\section{Study design}

This was a single-center, randomized, prospective clinical trial and was performed in 6 ICUs in West China Hospital of Sichuan University. Randomisation was done by means of a computer-based random number table procedure, by staff not involved in treatment of the patients. Patients and therapists were not masked to the treatment. Patients data collection and analysis were masked to the treatment group allocation staff. The study was approved by the Ethics Committees of West China Hospital of Sichuan 
University (2017 - 102) and was registered at Chinese Clinical Trial Registry (www.chictr.org.cn, ChiCTRIPR-17012629). Informed consents were obtained from patients or patients' legal representatives.

\section{Patients}

Sixty-four critically ill patients (49 males and 15 females) with AKI or chronic kidney disease (CKD) receiving CRRT during September 2017 and September 2019 were successfully enrolled in this study. All the included participants who agreed with this study were randomly assigned to RCA-based CRRT with calcium-containing replacement solution (calcium-containing group, $n=35$ ) or the calcium-free replacement solution (calcium-free group, $n=29$ ).

\section{Inclusion and exclusion criteria}

The inclusion criteria were as follows: 1) Critically ill patients who need renal replacement therapy, such as AKI, ESRD or CKD, acute drug or pesticide intoxication patients, etc.; 2) Hemodynamically stable and suitability for CRRT with continuous venovenous hemodiafiltration (CVVHDF) modality; 3) Age between 18 to 80 years old; 4) Informed consent was given after enrolment. Patients were excluded for the study if they fulfilled these exclusion criteria: 1) Patients with citrate anticoagulant contraindications, such as sever liver failure, irreversible hypotension or hypoxemia; 2) Organ transplant patients; 3) Expected stay in ICU less than 24 hours; 4) Pregnant or breastfeeding; 5) Patients who cannot cooperate to the study because of mental diseases or other reasons.

\section{Intervention}

Prismaflex (Baxter (China) Investment Co., Ltd, Shanghai) CRRT machine, AN69 ST150 hemofilters (Baxter (China) Investment Co., Ltd, Shanghai) were applied for the extracorporeal circuit system. The AN69 ST150 set was replaced every 72 hours regularly. Patients in both groups were treated with CVVHDF. Vascular access was provided by the insertion of a double lumen catheter into either the femoral vein (Baxter International Inc, GDHK-1325, 13Fr, $250 \mathrm{~mm}$ ) or internal jugular vein (Baxter International Inc, GDHK-1215, 12Fr, $150 \mathrm{~mm}$ ). Post-dilution mode was used and the CRRT dose was 20$35 \mathrm{ml} / \mathrm{kg} / \mathrm{h}$ (dialysis: replacement fluid =1:1). The blood flow was set as $150 \mathrm{~mL} / \mathrm{min}$ and adjusted according to ionized calcium (iCa) concentration during treatment. Blood gas were tested by using a point-of-care blood gas analyzer (Cobas b123, Roche Diagnostics, Basel, Switzerland) at the beginning ( 0.5 hours, pre-filter), 2 hours and every 6 hours (pre- and post-filter) after starting CVVHDF. $4 \%$ trisodium citrate solution (200 mL, Qingshan Likang Pharmaceutical Co. Ltd., Chengdu, China) was pumped into the arterial line of the extracorporeal circuit at an initial rate of $170 \mathrm{~mL} / \mathrm{h}$ in both groups, and was titrated at increment or decrement of $10 \mathrm{~mL} / \mathrm{h}$ to achieve a target post-filter iCa level of $0.25-0.35 \mathrm{mmol} / \mathrm{L}$ and pre-filter iCa level of $0.90-1.20 \mathrm{mmol} / \mathrm{L}$. 
The commercial calcium-containing replacement solution (Qingshan Likang, Pharmaceutical Co. Ltd., Chengdu, China), which contained solution A (Glucose $10.6 \mathrm{mmol} / \mathrm{L}, \mathrm{Cl}^{-} 118 \mathrm{mmol} / \mathrm{L}, \mathrm{Mg}^{2+}$ $0.797 \mathrm{mmol} / \mathrm{L}, \mathrm{Ca}^{2+} 1.60 \mathrm{mmol} / \mathrm{L}$, and $\left.\mathrm{Na}^{+} 113 \mathrm{mmol} / \mathrm{L}\right)$ and solution $\mathrm{B}$ ( $5 \%$ sodium bicarbonate), was adopted in calcium-containing group. Two liters of solution A combined with $125 \mathrm{~mL}$ of solution $B$ had a $\mathrm{pH}$ of 7.40 and contained glucose $10.0 \mathrm{mmol} / \mathrm{L}, \mathrm{Cl}^{-} 110 \mathrm{mmol} / \mathrm{L}, \mathrm{Mg}^{2+} 0.75 \mathrm{mmol} / \mathrm{L}, \mathrm{Ca}^{2+} 1.50 \mathrm{mmol} / \mathrm{L}$, $\mathrm{Na}^{+} 141 \mathrm{mmol} / \mathrm{L}$, and $\mathrm{HCO}^{3-} 35 \mathrm{mmol} / \mathrm{L}$. During RCA based CVVHDF, relatively small dose (exp. solution A/B: $2 \mathrm{~L} / 40 \mathrm{~mL}$ ) of $5 \%$ sodium bicarbonate (solution $\mathrm{B}$ ) was delivered alone by a pump pre-filter; this dose could be adjusted at any time to correct for metabolic acidosis or alkalosis. $10 \%$ potassium chloride was also infused by was delivered by another pump pre-filter and the infusion dose as well as net ultrafiltration were adjusted according to the blood gas analysis results and clinical condition of the patient. $10 \%$ calcium gluconate was infused on an as-needed basis to maintain the target ionized calcium level as an intermittent intravenous bolus. Calcium-free replacement solution (Qingshan Likang, Pharmaceutical Co. Ltd., Chengdu, China), which contained solution A (Glucose $10.6 \mathrm{mmol} / \mathrm{L}, \mathrm{Cl}^{-}$ $115 \mathrm{mmol} / \mathrm{L}, \mathrm{Mg}^{2+} 0.797 \mathrm{mmol} / \mathrm{L}, \mathrm{Ca}^{2+} 0.0 \mathrm{mmol} / \mathrm{L}$, and $\mathrm{Na}^{+} 113 \mathrm{mmol} / \mathrm{L}$ ) and solution $\mathrm{B}$ (5\% sodium bicarbonate), was used in calcium-free group. In order to replace calcium losses in blood, intravenous $10 \%$ calcium gluconate was administered continuously by a pump via a T-branch pipe in post-filter at a speed of $18-25 \mathrm{~mL} / \mathrm{h}$. Other preset parameters were in accordance with calcium containing group. The standard scheme for application of these two replacement solutions in RCA-based post-dilution CVVHDF is shown in Fig. 1.

The decisions to start or stop CRRT were carried out by clinicians in both ICU and Nephrology department. CRRT was delivered according to manufacturer's specifications, including routinely circuit changes after 72 hours of use without clotting. CRRT was prescribed by nephrologists and delivered by nursing staff. Patients and treatment information was collected at a later time.

\section{Adverse reactions}

One of the most feared complication of patients in both groups during RCA-based CRRT was citrate accumulation. Hypocalcemia, metabolic acidosis and increased serum lactate levels were noticeable signs. An increased total/ionized calcium $\left(\mathrm{Ca} / \mathrm{Ca}^{2+}\right)$ ratio $(>2.5)$ was recognized as indicative of significant accumulation [18]. When citrate accumulation was suspected, the net citrate load administered to the patient would be decreased. RCA would be replaced by alternative circuit anticoagulation if irreversible citrate accumulation happened. Other adverse potential complications such as bleeding, hypernatremia or hyponatremia, metabolic alkalosis, paresthesia, twitching movement and cardiac dysrhythmia were recorded in both groups.

\section{Study endpoints}


The primary outcome was circuit lifespan (measured in hours). Circuit lifespan started from extracorporeal circulation of blood and ended when the circuit was clotting. The clotted circuit was characterized by having exceeded transmembrane pressure (TMP) over than $250 \mathrm{~mm} \mathrm{Hg}$ and/or visible clotting in air-trap chamber. CRRT circuits might be stopped without clotting because of the following reasons: 1) used for $72 \mathrm{~h}$ (without clotting); 2) catheter dysfunction and a new vascular access needed to be established (defined as unclear); 3 ) stopped for treatment reasons like operations and hospital transfer (defined as unclear); 4) patient relinquished CRRT (defined as unclear). Taken together, there were three possible outcomes for the circuits: clotted, without clotting or unclear. Clotted and "without clotting" circuits were defined as circuits with clear outcome.

The secondary endpoints included hospital mortality, length of ICU stays, kidney function recovery rate, complication incidence, as well as serum and effluent citrate and calcium concentration. Kidney function recovery was defined as normal serum creatinine $(<110 \mathrm{mmol} / \mathrm{L})$ and urine output $>800 \mathrm{ml} /$ day without diuretic applied according to previous studies $[19,20]$.

\section{Sample Size}

Sample size estimation was based on our previous circuit lifespan data of simplified RCA-based CRRT protocol. One hundred forty-eight sessions were performed and the mean hemofilter survival was $61.3 \pm$ $21.6 \mathrm{~h}$ [14]. The study was designed to demonstrate $\mathrm{a} \leq 20 \%$ difference in circuit lifespan with a 2-sided type I error of 0.05 and $80 \%$ power. According to the formula for calculating sample size of equivalent clinical trials [21], we set out to enroll 150 circuits (75 assigned to each group) to allow for a dropout rate of $15 \%$. As the study was prematurely discontinued due to slow enrolment of patients, 149 circuits were included at the end of the study (Fig. 2).

\section{Data collection}

Demographic data was collected when CRRT initiated and treatment group allocation was masked to data collection staff. We gathered clinical information, the need for mechanical ventilation and vasopressors, sequential organ failure assessment (SOFA) score, and the following laboratory variables when starting CRRT: serum urea, creatinine, estimate glomerular filtration rate (eGFR), hemoglobin, platelet count, prothrombin time (PT), activated partial thromboplastin time (APTT), alanine aminotransferase (ALT), aspartate aminotransferase (AST) and total bilirubin levels. Other information during CRRT was recorded, such as blood flow rate, the amount of $4 \%$ trisodium citrate solution and $10 \%$ calcium gluconate administrated, filtration fraction, dialysate and replacement solution infusion rates, catheter sites, initial and withdraw TMP, circuit survival, causes of circuit replacement and adverse reactions. Other vital signs of the patients were also monitored regularly. The levels of serum and effluent total calcium ( $\mathrm{tCa}$ ) were measured by Roche Cobas c702 analyzer (Roche Diagnostics, Basel, Switzerland) in both groups and analyzed at the beginning ( 0.5 hours), 6 hours, 12 hours and then every 
24 hours after CVVHDF. By the end of study, patients' information about length of ICU stay, kidney function recovery and causes of death were collected.

\section{Statistical analyses}

SPSS (IBM SPSS Statistics 19.0, Chicago, IL, USA) was applied for all statistical analyses. Continuous numeric variables showed normal distributions were compared by t-test and presented as mean \pm standard deviation (SD); Mann-Whitney $U$ test and median (interquartile range, IQR) was used for nonnormal distribution statistics. Categorical variables were analyzed by Pearson $\chi 2$ test or Fisher's exact test and presented as the frequency and proportion. The Kaplan-Meier survival analysis using the logrank test (Mantel-Cox test) was implemented for comparing the hemofilter survival within the two groups. Statistical significance was assigned to $P$ values of $<0.05$.

\section{Results}

\section{Participants and Recruitment}

From September 2017 to September 2019, a total of 115 patients were referred to this study while 9 patients did not meet eligibility criteria and 34 declined study participation. Seventy-one patients were randomized into two groups and 7 of them withdraw from study because of treatment modality changed from CRRT into intermittent hemodialysis (IHD, 3 patients), receiving CVVHDF less than 24 hours (3 patients) or discharged form hospital within 24 hours (1 patient). Finally, there were 35 patients and 82 circuits which were successfully included in calcium-containing replacement solution group, 29 patients and 67 filters in calcium-free group (see Fig. 2).

The primary diseases and main diagnosis of these patients were respiratory failure $(n=17)$, circulatory failure $(n=12)$, septic shock ( $n=11)$, postoperative $(n=9)$ and severe acute pancreatitis $(n=15)$. All of these patients were diagnosed with AKI or CKD (50/14). Demographic and clinical characteristics at the time of randomization were similar between groups and were listed in Table 1. The severity of critical illness was comparable between calcium-containing and calcium-free group by using the ICU scoring systems SOFA score. The groups were well matched at baseline with renal, liver and other hematological laboratory variables. 
Table 1

Demographic and Clinical Characteristics of the patients.

\begin{tabular}{|c|c|c|c|}
\hline Variable & $\begin{array}{l}\text { Calcium-containing group } \\
(n=35)\end{array}$ & $\begin{array}{l}\text { Calcium-free } \\
\text { group } \\
(n=29)\end{array}$ & $P$-value \\
\hline Age, years (SD) & $56.1(16.3)$ & $58.7(13.6)$ & 0.51 \\
\hline Male gender, $n$ /total (\%) & 28/35 (80.0) & $21 / 29(72.4)$ & 0.48 \\
\hline $\mathrm{BMI}, \mathrm{kg} / \mathrm{m}^{2}$ (SD) & $24.87(4.88)$ & $23.95(4.42)$ & 0.64 \\
\hline \multicolumn{4}{|l|}{ Main reason for admission to ICU, $n$} \\
\hline Respiratory failure & 8 & 9 & \multirow[t]{5}{*}{0.91} \\
\hline Circulatory failure & 7 & 5 & \\
\hline Septic shock & 6 & 5 & \\
\hline Postoperative & 6 & 3 & \\
\hline Severe acute pancreatitis & 8 & 7 & \\
\hline \multicolumn{4}{|l|}{ Reasons for CRRT, $n$} \\
\hline AKI & 26 & 24 & \multirow[t]{2}{*}{0.41} \\
\hline CKD & 9 & 5 & \\
\hline SOFA score, mean (SD) & $11(3)$ & $12(3)$ & 0.31 \\
\hline Mechanical ventilation, $n /$ total (\%) & $27 / 35(77.1)$ & $20 / 29(69.0)$ & 0.46 \\
\hline Vasopressor dependency, $n /$ total (\%) & $11 / 35(31.4)$ & $11 / 29(37.9)$ & 0.59 \\
\hline \multicolumn{4}{|l|}{ Renal variables, mean (SD) } \\
\hline Urea $(\mathrm{mmol} / \mathrm{L})$ & $17.6(8.4)$ & $21.8(8.6)$ & 0.14 \\
\hline Creatinine $(\mu \mathrm{mol} / \mathrm{L})$ & $291.5(88.6)$ & $324.5(168.4)$ & 0.49 \\
\hline $\operatorname{eGFR}\left(\mathrm{ml} / \mathrm{min} / 1.73 \mathrm{~m}^{2}\right)^{\star}$ & $19.66(13.05)$ & $16.11(19.99)$ & 0.27 \\
\hline
\end{tabular}

Abbreviations: SD, standard deviation; BMI, body mass index; ICU, intensive care unit; CRRT, continuous renal replacement therapy; AKI, acute kidney injury; CKD, chronic kidney disease; SOFA score, sequential organ failure assessment score; eGFR, estimate glomerular filtration rate; APTT, activated partial thromboplastin time; PT, prothrombin time; ALT, alanine aminotransferase; AST, aspartate aminotransferase.

*, median (interquartile range). 


\begin{tabular}{|c|c|c|c|}
\hline Variable & $\begin{array}{l}\text { Calcium-containing group } \\
(n=35)\end{array}$ & $\begin{array}{l}\text { Calcium-free } \\
\text { group } \\
(n=29)\end{array}$ & $P$-value \\
\hline Hemoglobin (g/L) & $88.0(17.9)$ & $92.2(21.7)$ & 0.48 \\
\hline Platelet count $\left(\times 10^{\wedge} 9 / \mathrm{L}\right)^{*}$ & $113.5(82.8)$ & $75.0(99.0)$ & 0.08 \\
\hline APTT (s)* & $38.0(20.3)$ & $35.1(12.2)$ & 0.99 \\
\hline $\mathrm{PT}(\mathrm{s})^{\star}$ & $13.4(3.6)$ & $15.7(4.6)$ & 0.13 \\
\hline \multicolumn{4}{|l|}{ Liver variables, mean (SD) } \\
\hline $\operatorname{ALT}(I U / L) *$ & $14(30)$ & $20(44)$ & 0.72 \\
\hline AST (IU/L)* & $40(31)$ & $46(46)$ & 0.61 \\
\hline Total bilirubin $(\mu \mathrm{mol} / \mathrm{L}) *$ & $15.1(14.2)$ & $18.7(15.1)$ & 0.15 \\
\hline \multicolumn{4}{|c|}{$\begin{array}{l}\text { Abbreviations: SD, standard deviation; BMI, body mass index; ICU, intensive care unit; CRRT, } \\
\text { continuous renal replacement therapy; AKI, acute kidney injury; CKD, chronic kidney disease; SOFA } \\
\text { score, sequential organ failure assessment score; eGFR, estimate glomerular filtration rate; APTT, } \\
\text { activated partial thromboplastin time; PT, prothrombin time; ALT, alanine aminotransferase; AST, } \\
\text { aspartate aminotransferase. }\end{array}$} \\
\hline \multicolumn{4}{|c|}{ *, median (interquartile range). } \\
\hline
\end{tabular}

\section{Treatment and metabolic parameters during CVVHDF}

Treatment parameters during CVVHDF were comparable and summarized in Table 2. The infusion rate of dialysate solutions $A$ was $1.07 \pm 0.22$ in calcium-containing group and $1.03 \pm 0.13 \mathrm{~L} / \mathrm{h}$ in calcium-free group, while rates of post-filter replacement solutions $A$ were $0.98 \pm 0.13$ and $0.91 \pm 0.19 \mathrm{~L} / \mathrm{h}$, respectively. Mean filtration fraction was set up to nearly $20 \%$. The blood flow rate $(143.0 \pm 9.5 \mathrm{vs} 144.7 \pm 6.0 \mathrm{~mL} / \mathrm{h}, P$ $=0.28)$ and $4 \%$ trisodium citrate infusion rates $(171.1 \pm 15.9$ vs $169.0 \pm 15.1 \mathrm{~mL} / \mathrm{h}, P=0.49)$ were adjusted to maintain their post-filter ionized calcium level within $0.25-0.35 \mathrm{mmol} / \mathrm{L}$. Total $10 \%$ calcium gluconate infusion dose was set up according to serum ionized calcium concentration (target range 0.90-1.20 mmol/L). In calcium-containing group, it was infused as an intermittent intravenous bolus when the pre-filter ionized serum calcium level was below $0.85 \mathrm{mmol} / \mathrm{L}$ and the average infusion dose was $1.47 \pm 1.34 \mathrm{~mL} / \mathrm{h}(0.34 \pm 0.31 \mathrm{mmol} / \mathrm{h})$. In calcium-free group, intravenous $10 \%$ calcium gluconate was administered continuously by a pump and the mean infusion rate was $20.16 \pm 3.62 \mathrm{~mL} / \mathrm{h}(4.69 \pm$ $0.84 \mathrm{mmol} / \mathrm{h}, P<0.001$ ). Median initial TMP were 53.0 (IQR 16.5) and 55.0 (IQR 30.0) $\mathrm{mmHg}$, and median TMP when withdrawing circuits were 110.0 (IQR 127.5) verses 127.0 (IQR 193.0) mmHg, respectively. 
Table 2

Treatment parameters during CVVHDF of the two groups.

\begin{tabular}{|llll|}
\hline Parameters, mean (SD) & Calcium-containing group & $\begin{array}{l}\text { Calcium-free } \\
\text { group }\end{array}$ & P-value \\
\hline Vascular site & & & \\
\hline Femoral vein, $n$ /total & $31 / 35$ & $27 / 29$ & 0.68 \\
\hline Right Jugular vein, $n$ /total & $4 / 35$ & $2 / 29$ & \\
\hline Dialysate rate (L/h) & $1.07(0.22)$ & $1.03(0.13)$ & 0.16 \\
\hline Post-filter replacement fluid rate (L/h) & $0.98(0.13)$ & $0.91(0.19)$ & 0.73 \\
\hline Filtration fraction (\%) & $19.3(2.5)$ & $19.7(2.3)$ & 0.54 \\
\hline Blood flow rate (mL/min) & $143.0(9.5)$ & $144.7(6.0)$ & 0.28 \\
\hline 4\% trisodium citrate infusion rate (mL/h) & $171.1(15.9)$ & $169.0(15.1)$ & 0.49 \\
\hline 10\% calcium gluconate supplement (mL/h) & $1.47(1.34)$ & $20.16(3.62)$ & $<0.001$ \\
\hline Initial TMP (mmHg)* & $53.0(16.5)$ & $55.0(30.0)$ & 0.50 \\
\hline Withdraw TMP (mmHg)* & $110.0(127.5)$ & $127.0(193.0)$ & 0.65 \\
\hline Abbreviations: SD, standard deviation; TMP, transmembrane pressure. & & \\
\hline SD, standard deviation; * Median (Interquartile range, IQR) & & \\
\hline
\end{tabular}

\section{Circuit lifespan and calcium concentration}

Total 149 circuits (82 in calcium-containing group and 67 in calcium-free group) and 7609 circuit hours (4335 versus 3274 hours) were included. Total circuit lifespans had no significant difference between calcium-containing and calcium-free group. Mean circuit lifespan was 58.1 hours (95\% $\mathrm{Cl} 53.8-62.4)$ in calcium-containing group versus 55.3 hours $(95 \% \mathrm{Cl} 49.7-60.9$, log rank $P=0.89)$ in calcium-free group. The Kaplan-Meier survival analysis revealed that the circuit lifespan was similar using the calciumcontaining replacement solution compared with that in the calcium-free group (Fig. 3a). Total circuits outcomes and reasons for circuits disconnection were summarized in Table 3. 
Table 3

The circuits and patients' clinical outcomes.

\begin{tabular}{|c|c|c|c|}
\hline Variable & Calcium-containing Group & $\begin{array}{l}\text { Calcium-free } \\
\text { Group }\end{array}$ & $P$-value \\
\hline Total circuits outcomes & $\mathrm{n}=82$ & $n=67$ & \\
\hline Mean survival time (95\% Cl, hours) & $58.1(53.8-62.4)$ & $55.3(49.7-60.9)$ & 0.89 \\
\hline Median (Range, hours) & $55.3(5.3,72.0)$ & $53.0(5.5,72.0)$ & \\
\hline$\geq 24$ hours & $86.2 \%$ & $83.6 \%$ & \\
\hline$\geq 48$ hours & $56.1 \%$ & $55.2 \%$ & \\
\hline$\geq 72$ hours & $32.9 \%$ & $29.2 \%$ & \\
\hline \multicolumn{4}{|c|}{ Reasons for circuits disconnection, n/total (\%) } \\
\hline Circuit clotting & $38(46.3 \%)$ & $28(41.8 \%)$ & \multirow[t]{6}{*}{0.57} \\
\hline Air-trap chamber clotting & $8 / 38$ & $3 / 28$ & \\
\hline Regular filter replacement (72 hours) & $24(29.3 \%)$ & $20(29.9 \%)$ & \\
\hline Catheter dysfunction & $0(0.0 \%)$ & $2(3.2 \%)$ & \\
\hline Relinquishment of CVVHDF & $15(18.3 \%)$ & $14(20.9 \%)$ & \\
\hline Transport & $5(6.1 \%)$ & $3(4.5 \%)$ & \\
\hline \multicolumn{4}{|l|}{ Summarized total circuits outcomes } \\
\hline Clotted & $38(46.3 \%)$ & $28(41.8 \%)$ & \multirow[t]{3}{*}{0.82} \\
\hline Without clotting (72 hours) & $24(29.3 \%)$ & $20(29.9 \%)$ & \\
\hline Unclear & $20(24.4 \%)$ & $19(28.4 \%)$ & \\
\hline First circuits outcomes & $n=35$ & $n=29$ & \\
\hline Mean survival time (95\% Cl, hours) & $62.3(57.3-67.3)$ & $58.3(50.1-66.6)$ & 0.99 \\
\hline \multicolumn{4}{|c|}{ Reasons for circuits disconnection, n/total (\%) } \\
\hline Circuit clotting & $16(45.7 \%)$ & $12(41.4 \%)$ & \multirow[t]{3}{*}{0.76} \\
\hline Air-trap chamber clotting & $2 / 16$ & $2 / 12$ & \\
\hline Regular filter replacement (72 hours) & $13(37.1 \%)$ & $9(31.0 \%)$ & \\
\hline \multicolumn{4}{|c|}{$\begin{array}{l}\text { Abbreviations: } \mathrm{Cl} \text {, confidence interval; CVVHDF, continuous venovenous hemodiafiltration, ICU, } \\
\text { intensive care unit. }\end{array}$} \\
\hline * Median (Interquartile range) & & & \\
\hline
\end{tabular}




\begin{tabular}{|llll|}
\hline Variable & Calcium-containing Group & $\begin{array}{l}\text { Calcium-free } \\
\text { Group }\end{array}$ & P-value \\
\hline Catheter dysfunction & $0(0.0 \%)$ & $1(3.4 \%)$ & \\
\hline Relinquishment of CVVHDF & $4(11.4 \%)$ & $5(17.2 \%)$ & \\
\hline Transport & $2(5.7 \%)$ & $2(6.9 \%)$ & \\
\hline Patients outcomes & $\mathrm{n}=35$ & $\mathrm{n}=29$ & 0.70 \\
\hline Hospital Mortality & $40.0 \%(14 / 35)$ & $44.8 \%(13 / 29)$ & 0.63 \\
\hline Kidney recovery & $54.3 \%(19 / 35)$ & $48.3 \%(14 / 29)$ & 0.27 \\
\hline ICU stays (days)* & $20(27)$ & $26(15)$ & 0.81 \\
\hline Adverse events (\%) & & $3 / 29(10.3)$ & 0.55 \\
\hline Citrate accumulation & $3 / 35(8.6)$ & $0 / 29$ & 0.96 \\
\hline Bleeding & $1 / 35(2.9)$ & $4 / 29(13.8)$ & \\
\hline Hyponatremia & $5 / 35(14.3)$ & & \\
\hline $\begin{array}{l}\text { Abbreviations: Cl, confidence interval; CVVHDF, continuous venovenous hemodiafiltration, ICU, } \\
\text { intensive care unit. }\end{array}$ & & & \\
\hline * Median (Interquartile range) & & & \\
\hline
\end{tabular}

Survival of the first circuit were also comparable between RCA with calcium-containing and calcium-free replacement solution (62.3 (95\% $\mathrm{Cl} 57.3-67.3)$ versus $58.3(95 \% \mathrm{Cl} 50.1-66.6)$ hours, log rank $P=0.99$; Fig. 3b). Reasons for circuit disconnection of the first circuit were also presented in Table 3 . Sixteen of 35 first circuits $(45.7 \%, 2$ air-trap chamber clotting) in the calcium-containing group were stopped due to clotting, compared with 12 of 29 (41.4\%, 2 air-trap chamber clotting) in the calcium-free group. There were 22 first circuits $(13 / 35,37.1 \%$ versus $9 / 29,31.0 \%, P=0.61)$ had normal function within 72 hours and were regularly changed. One patient in calcium-free group had catheter dysfunction and a new vascular access needed to be established together with a new filter changed. Other first circuits were disconnected due to relinquishment of CVVHDF $(4 / 35,11.4 \%$ versus $5 / 29,17.2 \%)$ or transport $(2 / 35,5.7 \%$ versus $2 / 29$, $6.9 \%)$.

To further investigate lifespans of circuits with clear outcomes, we excluded "unclear" outcome and analyzed "clotted" or "without clotting" filters. Sixty-two in calcium-containing group and 50 circuits in calcium-free group were included. The Kaplan-Meier survival analysis also showed no significant difference in circuit life between groups (mean 55.2 versus 51.2 hours, $P=0.70$, see Supplementary Fig. 1). 
During RCA-CVVHDF, total calcium (tCa) concentration in serum and effluent, as well as serum and postfilter ionized calcium (iCa), were summarized in Table 4. Both the systemic serum tCa and iCa concentration were similar at the initial time and turned slightly lower in calcium-containing group in the first 48 hours during CVVHDF $(P<0.05$, see Table 4). However, after 72 hours of treatment, the systemic serum tCa and iCa levels in calcium-containing group gradually increased and became comparable with calcium-free group $(2.06 \pm 0.22$ versus $2.15 \pm 0.22 \mathrm{mmol} / \mathrm{L}, P=0.37 ; 0.93 \pm 0.07$ versus $1.00 \pm$ $0.08 \mathrm{mmol} / \mathrm{L}, P=0.002$, respectively). The effluent tCa showed similar increasing trend with serum tCa (see Table 4). Interestingly, as post-dilution of calcium-containing replacement solution, the post-filter iCa concentration was elevated in calcium-containing group and post-dilution of calcium-free replacement solution made the post-filter iCa lower $(P<0.05)$. Both groups achieved the target range of the mean postfilter iCa levels (from $0.25 \pm 0.10$ to $0.33 \pm 0.07 \mathrm{mmol} / \mathrm{L}$, see Table 4). 
Table 4

Calcium concentration in patients during treatment with RCA-based CVVHDF.

\begin{tabular}{|c|c|c|c|c|c|c|c|}
\hline \multirow[t]{2}{*}{ Time } & & \multicolumn{3}{|c|}{ Serum Calcium (mmol/L) } & \multicolumn{3}{|c|}{ Effluent/Post-filter Calcium (mmol/L) } \\
\hline & & $\begin{array}{l}\text { Calcium- } \\
\text { containing } \\
\text { Group }\end{array}$ & $\begin{array}{l}\text { Calcium- } \\
\text { free Group }\end{array}$ & $\begin{array}{l}P \text { - } \\
\text { value }\end{array}$ & $\begin{array}{l}\text { Calcium- } \\
\text { containing } \\
\text { Group }\end{array}$ & $\begin{array}{l}\text { Calcium- } \\
\text { free Group }\end{array}$ & $\begin{array}{l}P- \\
\text { value }\end{array}$ \\
\hline \multirow{2}{*}{$\begin{array}{l}0.5 \\
\text { hours }\end{array}$} & $\mathrm{tCa}^{*}$ & $1.96 \pm 0.20$ & $2.05 \pm 0.31$ & 0.23 & $1.44+0.37$ & $1.62 \pm 0.37$ & 0.03 \\
\hline & $\mathrm{iCa}^{\#}$ & $0.94 \pm 0.14$ & $1.00 \pm 0.14$ & 0.23 & $0.33 \pm 0.06$ & $0.27 \pm 0.11$ & 0.31 \\
\hline \multirow[t]{2}{*}{$\begin{array}{l}6 \\
\text { hours }\end{array}$} & $\mathrm{tCa}$ & $1.94 \pm 0.22$ & $2.19 \pm 0.28$ & $\begin{array}{l}< \\
0.001\end{array}$ & $1.47 \pm 0.40$ & $1.65 \pm 0.36$ & 0.11 \\
\hline & $\mathrm{iCa}$ & $0.90 \pm 0.10$ & $0.95 \pm 0.15$ & 0.06 & $0.33 \pm 0.07$ & $0.28 \pm 0.16$ & 0.01 \\
\hline \multirow{2}{*}{$\begin{array}{l}12 \\
\text { hours }\end{array}$} & $\mathrm{tCa}$ & $1.94 \pm 0.33$ & $2.22 \pm 0.28$ & 0.003 & $1.49 \pm 0.37$ & $1.73 \pm 0.26$ & 0.02 \\
\hline & $\mathrm{iCa}$ & $0.89 \pm 0.10$ & $1.00 \pm 0.16$ & $<.001$ & $0.32 \pm 0.07$ & $0.29 \pm 0.14$ & 0.21 \\
\hline \multirow{2}{*}{$\begin{array}{l}24 \\
\text { hours }\end{array}$} & $\mathrm{tCa}$ & $2.02 \pm 0.19$ & $2.27 \pm 0.28$ & 0.001 & $1.54 \pm 0.38$ & $1.74 \pm 0.41$ & 0.08 \\
\hline & $\mathrm{iCa}$ & $0.91 \pm 0.10$ & $1.04 \pm 0.13$ & $<.001$ & $0.32 \pm 0.07$ & $0.30 \pm 0.12$ & 0.44 \\
\hline \multirow{2}{*}{$\begin{array}{l}48 \\
\text { hours }\end{array}$} & $\mathrm{tCa}$ & $2.05 \pm 0.32$ & $2.29 \pm 0.33$ & 0.02 & $1.62 \pm 0.36$ & $1.89 \pm 0.23$ & 0.02 \\
\hline & $\mathrm{iCa}$ & $0.91 \pm 0.10$ & $1.01 \pm 0.12$ & $\begin{array}{l}< \\
0.001\end{array}$ & $0.32 \pm 0.07$ & $0.28 \pm 0.07$ & 0.01 \\
\hline \multirow{2}{*}{$\begin{array}{l}72 \\
\text { hours }\end{array}$} & $\mathrm{tCa}$ & $2.06 \pm 0.22$ & $2.15 \pm 0.22$ & 0.37 & $1.66 \pm 0.39$ & $1.80 \pm 0.26$ & 0.45 \\
\hline & $\mathrm{iCa}$ & $0.93 \pm 0.07$ & $1.00 \pm 0.08$ & 0.002 & $0.31 \pm 0.07$ & $0.25 \pm 0.10$ & 0.01 \\
\hline \multicolumn{8}{|c|}{$\begin{array}{l}\text { Abbreviations: RCA, regional citrate anticoagulation; CVVHDF, continuous venovenous } \\
\text { hemodiafiltration. }\end{array}$} \\
\hline \multicolumn{8}{|c|}{$\begin{array}{l}\text { *: tCa: Serum total calcium versus effluent total calcium, data were presented as mean } \pm \text { standard } \\
\text { deviation (SD). }\end{array}$} \\
\hline \multicolumn{8}{|c|}{ \#: iCa: Serum ionized calcium versus Post-filter ionized calcium, data were presented as mean \pm SD. } \\
\hline
\end{tabular}

\section{Secondary outcomes and adverse events}

Mortality rates were comparable between groups during hospitalization between groups: 14/35 calciumcontaining group patients $(40.0 \%)$ died versus $13 / 29$ patients $(44.8 \%)$ in the calcium-free group died ( $P=$ 0.70 , Table 3). Furthermore, there was no difference between groups in kidney function recovery rates, 
with $19 / 35$ patients $(54.3 \%)$ in the calcium-containing group versus $14 / 29$ patients $(48.3 \%)$ in the calcium-free group $(P=0.63)$ had normal kidney function after withdrawing CVVHDF. Length of ICU stays were also similar in calcium-containing and calcium-free group (median 20 versus 26 days, see Table 3 ).

Three patients (8.6\%) in calcium-containing group and $3(10.3 \%)$ in calcium-free group developed significant citrate accumulation. These patients showed significant systemic hypocalcemia $(<$ $0.8 \mathrm{mmol} / \mathrm{L})$ and increased total/ionized calcium $\left(\mathrm{Ca} / \mathrm{Ca}^{2+}\right)$ ratio $(>2.5)$, meanwhile, metabolic acidosis ( $\mathrm{pH}$ value $<7.20$, normal range $7.35-7.45)$ and increased serum lactate levels $(>5.0 \mathrm{mmol} / \mathrm{L}$, normal range 1.0-1.8 mmol/L) were observed. All of the abnormal index reverted by increasing of calcium supplementation and replacement of anticoagulation from RCA into low molecular weight heparin $(n=4)$ or no anticoagulation $(n=2)$. Only one patient in the calcium-containing group had bleeding at the femoral vein puncture site. Hyponatremia ( $130 \mathrm{mmol} / \mathrm{L}$ ) was seen in 9 patients (5/35 versus 4/29) and none of the patients had other severe complications like hypernatremia, metabolic alkalosis, paresthesia, twitching movement or cardiac dysrhythmia.

\section{Discussion}

\section{Key Findings}

In the present study, we found that simplified RCA using a calcium-containing replacement solution was safety and efficiency compared with calcium-free replacement in post-dilution CVVHDF. Compared to the calcium-free replacement solution, patients treated with calcium-containing solution had similar circuit lifespan as well as clinical outcomes in mortality, kidney function recovery, the length of the ICU stays and adverse events like citrate accumulation and hyponatremia rates. Systemic serum concentrations of tCa and iCa and post-filter iCa were slightly lower in calcium-containing group, whereas the calcium levels were gradually increased and became comparable between groups after 72 hours treatment.

\section{Relationship to Previous Studies}

CRRT has been frequently applied in critically ill patients with kidney dysfunction and the choice of extracorporeal anticoagulation is important. Compared to widely used heparin and low-molecular-weight heparin anticoagulation, RCA is more efficacious in prolonging circuit life span and reducing the risk of bleeding $[3,4,10,22]$.

During RCA-based CRRT, sodium citrate is infused through arterial blood in the extracorporeal circuit. Citrate and calcium react in the blood and form calcium citrate, and then ionized calcium in the blood significantly decrease so that the blood coagulation processes are blocked [7]. Since the citrate anions and calcium-citrate complexes have low molecular weight (approximately $300 \mathrm{Da}$ ) and a quantity of them can be discharged in the effluent fluid, systemic calcium infusion is critical to replace calcium losses and prevent hypocalcemia [6, 9]. Traditional RCA protocols apply calcium-free replacement solution in CRRT and calcium chloride or calcium gluconate should be infused at the end of the 
extracorporeal circuit or directly infused into the patient via a central venous line, in order to maintain a systemic iCa at the physiological range $[9,23]$. In recent years, the adoption of conventional calciumcontaining replacement fluids in RCA-based CRRT represents a further simplification that reduces the need for calcium infusion, as well as labor intensive and the risk of iatrogenic errors when calcium-free solutions are handled [24-26].

Cointault et al [16] developed a simplified method of RCA during CVVHDF, which used Anticoagulant Citrate Dextrose Formula A (ACD-A, Hospal, Lyon, France) solution containing $112.9 \mathrm{mmol} / \mathrm{l}$ disodium citrate (3.22\%) and reinjection replacement solution and dialysate composed of Hemosol B0 solution combined with Hemosol B0 5.88\% HCO3Na solution (calcium $1.75 \mathrm{mmol} / \mathrm{L}$, Hospal, Lyon, France). The loss of calcium through the dialyser was compensated by infusion of a calcium chloride solution $(1.37 \mathrm{mmol} / \mathrm{h})$ to maintain plasma iCa ${ }^{2+}>1.1 \mathrm{mmol} / \mathrm{l}$. Gupta et al [15] evaluated ACD-A (3\% solution: trisodium citrate, $2.2 \%$; and citric acid, $0.8 \% ; 112.9 \mathrm{mmol}$ of citrate/L; Baxter) solution in combination with a commercially available calcium-containing dialysate for regional anticoagulation in CVVHDF. A lowcalcium peritoneal dialysis solution (calcium $1.25 \mathrm{mmol} / \mathrm{L}$, Baxter Healthcare, Deerfield, IL) was used as dialysate, as well as isotonic saline was used as replacement fluid and infused proximal to the hemofilter. Calcium chloride ( $10 \%$ solution, $5.4 \pm 4.35 \mathrm{mmol} / \mathrm{d}$ ) was administered on an as-needed basis as an intermittent intravenous bolus every 6 hours to maintain systemic venous iCa levels at 0.88 to $1.0 \mathrm{mmol} / \mathrm{L}$. Despite all of these protocols used calcium-containing replacement solution or dialysate, lower bicarbonate concentration dialysates were infused to compensate the buffer overload due to the bicarbonate production ensuing from the net citrate load to the patient, therefore prevented metabolic alkalosis during RCA $[27,28]$. Customized replacement solution and dialysate dedicated for RCA-CRRT were still needed, which made it difficult to change the anticoagulation methods as the conditions of critical patients change rapidly. We developed a simplified protocol for RCA using a commercial calciumcontaining replacement solution for continuous venovenous hemofiltration (CVVH) in 2012 [14]. In our protocol, two liters of solution A combined with $125 \mathrm{~mL}$ of solution $B$ have a pH of 7.40 and contained $\mathrm{Ca}^{2+} 1.50 \mathrm{mmol} / \mathrm{L}$ and $\mathrm{HCO}^{3-} 35 \mathrm{mmol} / \mathrm{L}$. The solution $\mathrm{B}$ ( $5 \%$ sodium bicarbonate) was delivered alone by a pump pre-filter, which made it could be adjusted at any time to correct for metabolic acidosis or alkalosis. When the anticoagulation switched from RCA to low-molecular-weight heparin or no anticoagulation, the nurses only needed to withdraw $4 \%$ citrate and increased the blood flow and solution $B$ infusion rates. No other solution was needed to be replaced or discharged. Therefore, this calciumcontaining solution protocol can save medical resources, decrease the labor intensive, remarkably shorten the downtime and simplify the anticoagulation in CRRT.

It has been reported that RCA with calcium-containing replacement solution is compatible with an adequate filter lifespan without increasing the risk of venous drip chamber clotting $[24,26,29]$. However, to our best knowledge, this is the first RCT to compare the efficiency and safety between calciumcontaining and calcium-free replacement solution in post-dilution RCA-based CVVHDF. A systematic review reported that the mean hemofilter survival time is 55.9 (IQR 32.8 to 68.9) hours in RCA-CRRT [30]. Gupta et al [15] reported that mean circuit lifespan was $63.5 \pm 27.1$ hours and Cointault et al [16] found 
that mean filter survival was $39 \pm 11$ hours in RCA using calcium-containing replacement and dialysate solutions for CVVHDF. All of these circuit lifespans are comparable to the calcium-containing group in our study (mean 58.1 ( $95 \%$ Cl 53.8-62.4) hours). Interestingly, in our pilot, similar circuit lifespan (mean 55.3 (49.7-60.9) hours, log rank $P=0.89$ ) was observed in RCA with calcium-free replacement solution. Other factors which can affect circuit lifespan independent of anticoagulation such as CRRT modality, delivery dose, filtration fraction, vascular sites, blood flow rate and $4 \%$ sodium citrate infusion rate were balanced between groups. Some protocols use a fixed dose of citrate in relation to blood flow according to an algorithm, and target such doses at about $3 \mathrm{mmol}$ citrate/L blood flow [27]. Infusion rate of $4 \%$ trisodium citrate of our protocol was set up as $170 \mathrm{~mL} / \mathrm{h}$ and mean delivery blood flow rate was about $144 \mathrm{~mL} / \mathrm{min}$, which made the citrate level in the extracorporeal circuit maintaining approximately $2.88 \mathrm{mmol}$ citrate/L blood flow. Citrate dose was slightly lower in our study then previous described, but we still got acceptable circuit lifespan. Despite post-filter iCa concentration was higher in calcium-containing group during CVVHDF $(P<0.05)$, circuit lifespan as well as air-trap chamber clotting rates $(8 / 38$ versus $3 / 28, P$ $=0.27$ ) had no significant difference between groups. These results of our study demonstrated the efficiency of simplified regional citrate anticoagulation using a calcium-containing replacement solution in post-dilution CVVHDF.

In RCA based CRRT, calcium concentration is critical to be monitored to estimate the safety and efficacy of anticoagulation. According to previous studies, a lower than usual systemic ionized calcium level (0.9$1 \mathrm{mmol} / \mathrm{L}$ ) should be considered a reasonable target while performing RCA CRRT in the clinical scenario $[27,31]$. The post-filter circuit ionized calcium concentration is usually maintained $<0.3-0.5 \mathrm{mmol} / \mathrm{L}$ [27]. In our hospital, a target pre-filter ionized calcium level of $0.90-1.20 \mathrm{mmol} / \mathrm{L}$ and post-filter ionized calcium level of $0.25-0.35 \mathrm{mmol} / \mathrm{L}$ have been applied. Since the calcium-containing group patients only got $10 \%$ calcium gluconate infusion on an as-needed basis by intermittent intravenous bolus when the pre-filter ionized calcium level $<0.85 \mathrm{mmol} / \mathrm{L}$, the systemic pre-filter concentrations of serum tCa, iCa and effluent tCa were slightly lower than calcium-free group in the first 48 hours of CVVHDF. However, majority of the hypocalcemia could be corrected quickly after calcium gluconate administration $(0.34 \pm$ $0.31 \mathrm{mmol} / \mathrm{h}$ ) in calcium-containing group. This calcium infusion is lower than Cointault et al. (calcium chloride solution, $1.37 \mathrm{mmol} / \mathrm{h}$ ) [16] but slightly higher than Gupta et al. (calcium chloride solution, $5.40 \pm$ $4.35 \mathrm{mmol} / \mathrm{d}, 0.23 \pm 0.18 \mathrm{mmol} / \mathrm{h}$ ) [15]. In calcium-free group, the mean intravenous $10 \%$ calcium gluconate administration rate was $20.16 \pm 3.62 \mathrm{~mL} / \mathrm{h}(4.69 \pm 0.84 \mathrm{mmol} / \mathrm{h})$, which was comparable to calcium pump rate in RCA group of Schilder et al. [10]. Nevertheless, the appropriate calcium infusion amount still needed to be investigated in our future studies and clinical RCA based CRRT practice using calcium-containing replacement solution.

In this work, we included 64 patients and the overall hospital mortality had no significant difference between calcium-containing (40.0\%) and calcium-free group (44.8\%). Our findings were in concordance with previous studies and the mortality was similar with the citrate group patients $(41.3 \%, 183 / 443)$ in a recent systematic review [2]. Moreover, these two groups in our study had comparable length of ICU stays and kidney function recovery rates, which implied that calcium-containing replacement solution won't do harm to the patient-centered outcomes. As for adverse reactions, rates of citrate accumulation which lead 
to RCA withdrawal were comparable between groups (3 in each group, total 9.4\%) in our study and this frequency is similar to rate reported by Nurmohamed et al. (9\%) [25] and lower than pooled rate of citrate accumulation (12\%) in liver failure patients [30]. Furthermore, the amount of citrate delivered and the blood flow rates were similar between groups, all of them indicated that citrate overload and severe citrate accumulation rate did not differ between calcium-containing and calcium-free replacement solution. Only one patient (1/31) in the calcium-containing group had hemorrhage at the femoral vein puncture site, which might be caused by the high risk of bleeding since the patient had hemorrhagic pancreatitis. No other adverse complications were seen in either group. Patients in our study had rare adverse reactions and this is in concordance with previous studies [32]. Simplified regional citrate anticoagulation using a calcium-containing replacement solution, as well as calcium-free replacement solution are safe protocols for usual clinical practice.

Weaknesses of this trial include that this study is a single-center RCT and the sample size is relatively small, therefore, a larger sample capacity and a multi-center RCT are needed in the future research. Subsequently, there was a slight imbalance between groups in filters numbers because 6 patients in calcium-containing group had long CRRT duration time and used more than 10 filters per person, but there were no differences in patients' numbers between admission categories. What's more, we only used the Prismaflex® Baxter CRRT machine in this trial, application of our simplified RCA using a calciumcontaining replacement solution CRRT protocol need to be further confirmed on other devices like Fresenius $\mathrm{CiCa}{ }^{\circledR}$ and other CRRT modes.

\section{Conclusion}

In conclusion, patient received RCA based CRRT with calcium-containing or calcium-free replacement solution had similar mortality, kidney outcome and adverse events rates. Since our simplified RCA using a calcium-containing replacement solution protocol had similar and acceptable filter lifespan for postdilution CVVHDF, and obviated the need for a separate venous access for large dose of continuous intravenous calcium infusion, it is more convenient to be used in clinical practice.

\section{Abbreviations}

RCA, regional citrate anticoagulation; CRRT, continuous renal replacement therapy; CVVHDF, continuous venovenous hemodiafiltration; AKI, acute kidney injury; ESRD, end stage renal disease; CVVH, continuous venovenous hemofiltration; RCT, randomized clinical trial; CKD, chronic kidney disease; TMP, transmembrane pressure; SOFA score, sequential organ failure assessment score; eGFR, estimate glomerular filtration rate; $\mathrm{PT}$, prothrombin time; APTT, activated partial thromboplastin time; ALT, alanine aminotransferase; AST, aspartate aminotransferase; IQR, interquartile range.

\section{Declarations}

Acknowledgements 
None.

\section{Authors' contribution}

Tiantian Wei, Xin Tang, Ling Zhang*, Li Lin, Peiyun Li, Fang Wang, Ping Fu

L.Z., and P.F participated in research design. T.W., X.T., P.L., and F.W. conducted experiments and data collection. L.L. was in charge of patients randomisation. T.W. performed data analysis. T.W. and L.Z. wrote or contributed to the writing of the manuscript.

\section{Funding}

The study was supported by 1.3 .5 project for disciplines of excellence-Clinical Research Incubation Project, West China Hospital, Sichuan University (18HXFH018).

\section{Availability of data and materials}

Not applicable.

\section{Ethics approval and consent to participate}

The datasets used and/or analyzed during the current study are available from the corresponding author on reasonable request.

\section{Consent for publication}

Written informed consents were obtained from patients or patients' legal representatives for their data to be used for research and publication.

\section{Conflict of Interest Statement}

No conflicts of interest, financial or otherwise, are declared by the authors.

\section{References}

1. Villa G, Ricci Z, Ronco C. Renal Replacement Therapy. Crit Care Clin. 2015;31(4):839-848.

2. Liu C, Mao Z, Kang H, Hu J, Zhou F. Regional citrate versus heparin anticoagulation for continuous renal replacement therapy in critically ill patients: a meta-analysis with trial sequential analysis of randomized controlled trials. Crit Care. 2016;20(1):144.

3. Oudemans-van Straaten HM, Kellum JA, Bellomo R. Clinical review: anticoagulation for continuous renal replacement therapy--heparin or citrate? Crit Care. 2011;15(1):202.

4. Gattas DJ, Rajbhandari D, Bradford C, Buhr H, Lo S, Bellomo R. A Randomized Controlled Trial of Regional Citrate Versus Regional Heparin Anticoagulation for Continuous Renal Replacement Therapy in Critically III Adults. Crit Care Med. 2015;43(8):1622-1629. 
5. Kellum J, Lameire N, Aspelin P, Barsoum R, Burdmann E, Goldstein S, et al. Kidney disease: improving global outcomes (KDIGO) acute kidney injury work group. KDIGO clinical practice guideline for acute kidney injury[J]. Kidney Int Suppl (2011). 2012;2(1):1-138.

6. Abramson S, Niles JL. Anticoagulation in continuous renal replacement therapy. Curr Opin Nephrol Hypertens. 1999;8(6):701-707.

7. Oudemans-van Straaten HM, Ostermann M. Bench-to-bedside review: Citrate for continuous renal replacement therapy, from science to practice. Crit Care. 2012;16(6):249.

8. Palsson R, Niles JL. Regional citrate anticoagulation in continuous venovenous hemofiltration in critically ill patients with a high risk of bleeding. Kidney Int. 1999;55(5):1991-1997.

9. Tolwani AJ, Campbell RC, Schenk MB, Allon M, Warnock DG. Simplified citrate anticoagulation for continuous renal replacement therapy. Kidney Int. 2001;60(1):370-374.

10. Schilder L, Nurmohamed SA, Bosch FH, Purmer IM, den Boer SS, Kleppe CG, et al. Citrate anticoagulation versus systemic heparinisation in continuous venovenous hemofiltration in critically ill patients with acute kidney injury: a multi-center randomized clinical trial. Crit Care. 2014;18(4):472.

11. Ong SC, Wille KM, Speer R, Tolwani AJ. A continuous veno-venous hemofiltration protocol with anticoagulant citrate dextrose formula $A$ and a calcium-containing replacement fluid. Int $\mathrm{J}$ Artif Organs. 2014;37(6):499-502.

12. Kirwan CJ, Hutchison R, Ghabina S, Schwarze S, Beane A, Beane S, et al. Implementation of a Simplified Regional Citrate Anticoagulation Protocol for Post-Dilution Continuous Hemofiltration Using a Bicarbonate Buffered, Calcium Containing Replacement Solution. Blood Purif. 2016;42(4):349-355.

13. U.S. Food and Drug Administration. USFaDA. Current Drug Shortages Index. http://www.fda.gov/drugs/drugsafety/drugshortages/ucm050792.htm. (2013) Accessed 19 March 2014.

14. Zhang L, Liao Y, Xiang J, Qin W, Wu X, Tang Y, et al. Simplified regional citrate anticoagulation using a calcium-containing replacement solution for continuous venovenous hemofiltration. J Artif Organs. 2013;16(2):185-192.

15. Gupta M, Wadhwa NK, Bukovsky R. Regional citrate anticoagulation for continuous venovenous hemodiafiltration using calcium-containing dialysate. Am J Kidney Dis. 2004;43(1):67-73.

16. Cointault O, Kamar N, Bories P, Lavayssiere L, Angles O, Rostaing L, et al. Regional citrate anticoagulation in continuous venovenous haemodiafiltration using commercial solutions. Nephrol Dial Transplant. 2004;19(1):171-178.

17. Kirwan CJ, Hutchison R, Ghabina S, Schwarze S, Beane A, Ramsay S, et al. Implementation of a Simplified Regional Citrate Anticoagulation Protocol for Post-Dilution Continuous Hemofiltration Using a Bicarbonate Buffered, Calcium Containing Replacement Solution. Blood Purif. 2016;42(4):349-355.

18. Schneider AG, Journois $D$, Rimmelé T. Complications of regional citrate anticoagulation: accumulation or overload? Crit Care. 2017;21(1):281. 
19. Chen X, Chen Z, Wei T, Li P, Zhang L, Fu P. The Effect of Serum Neutrophil Gelatinase-Associated Lipocalin on the Discontinuation of Continuous Renal Replacement Therapy in Critically III Patients with Acute Kidney Injury. Blood Purif. 2019;48(1):10-17.

20. González Sanchidrián S, Deira Lorenzo JL, Muciño Bermejo MJ, Labrador Gómez PJ, Gómez-Martino Arroyo JR, Aresu S, et al. Survival and renal recovery after acute kidney injury requiring dialysis outside of intensive care units. Int Urol Nephrol. 2020;doi: 10.1007/s11255-020-02555-2.

21. Jones $B$, Jarvis $P$, Lewis JA, Ebbutt AF. Trials to assess equivalence: the importance of rigorous methods. BMJ. 1996;313(7048):36-39.

22. Meersch $M$, Küllmar M, Wempe $C$, Kindgen-Milles D, Kluge S, Slowinski T, et al. Regional citrate versus systemic heparin anticoagulation for continuous renal replacement therapy in critically ill patients with acute kidney injury (RICH) trial: study protocol for a multicentre, randomised controlled trial. BMJ open. 2019;9(1):e024411.

23. Durão MS, Monte JC, Batista MC, Oliveira M, lizuka IJ, Santos BF, et al. The use of regional citrate anticoagulation for continuous venovenous hemodiafiltration in acute kidney injury. Crit Care Med. 2008;36(11):3024-3029.

24. Morabito S, Pistolesi V, Tritapepe L, Zeppilli L, Polistena F, Strampelli E, et al. Regional citrate anticoagulation in cardiac surgery patients at high risk of bleeding: a continuous veno-venous hemofiltration protocol with a low concentration citrate solution. Crit Care. 2012;16(3):R111.

25. Nurmohamed SA, Jallah BP, Vervloet MG, Yldirim G, ter Wee PM, Groeneveld AB. Continuous venovenous haemofiltration with citrate-buffered replacement solution is safe and efficacious in patients with a bleeding tendency: a prospective observational study. BMC Nephrol. 2013;14:89.

26. Fiaccadori E, Pistolesi V, Mariano F, Mancini E, Canepari G, Inguaggiato P, et al. Regional citrate anticoagulation for renal replacement therapies in patients with acute kidney injury: a position statement of the Work Group "Renal Replacement Therapies in Critically III Patients" of the Italian Society of Nephrology. J Nephrol. 2015;28(2):151-164.

27. Morabito S, Pistolesi V, Tritapepe L, Fiaccadori E. Regional citrate anticoagulation for RRTs in critically ill patients with AKI. Clin J Am Soc Nephrol. 2014;9(12):2173-2188.

28. Morgera S, Schneider M, Slowinski T, Vargas-Hein O, Zuckermann-Becker $H$, Peters $H$, et al. A safe citrate anticoagulation protocol with variable treatment efficacy and excellent control of the acidbase status. Crit Care Med. 2009;37(6):2018-2024.

29. Morabito S, Pistolesi V, Tritapepe L, Vitaliano E, Zeppilli L, Polistena F, et al. Continuous veno-venous hemofiltration using a phosphate-containing replacement fluid in the setting of regional citrate anticoagulation. Int J Artif Organs. 2013;36(12):845-852.

30. Zhang W, Bai M, Yu Y, Li L, Zhao L, Sun S, et al. Safety and efficacy of regional citrate anticoagulation for continuous renal replacement therapy in liver failure patients: a systematic review and meta-analysis. Crit Care. 2019;23(1):22.

31. Davenport A, Tolwani A. Citrate anticoagulation for continuous renal replacement therapy (CRRT) in patients with acute kidney injury admitted to the intensive care unit. NDT Plus. 2009;2(6):439-447. 
32. Kindgen-Milles D, Brandenburger T, Dimski T. Regional citrate anticoagulation for continuous renal replacement therapy. Curr Opin Crit Care. 2018;24(6):450-454.

\section{Figures}

(A)

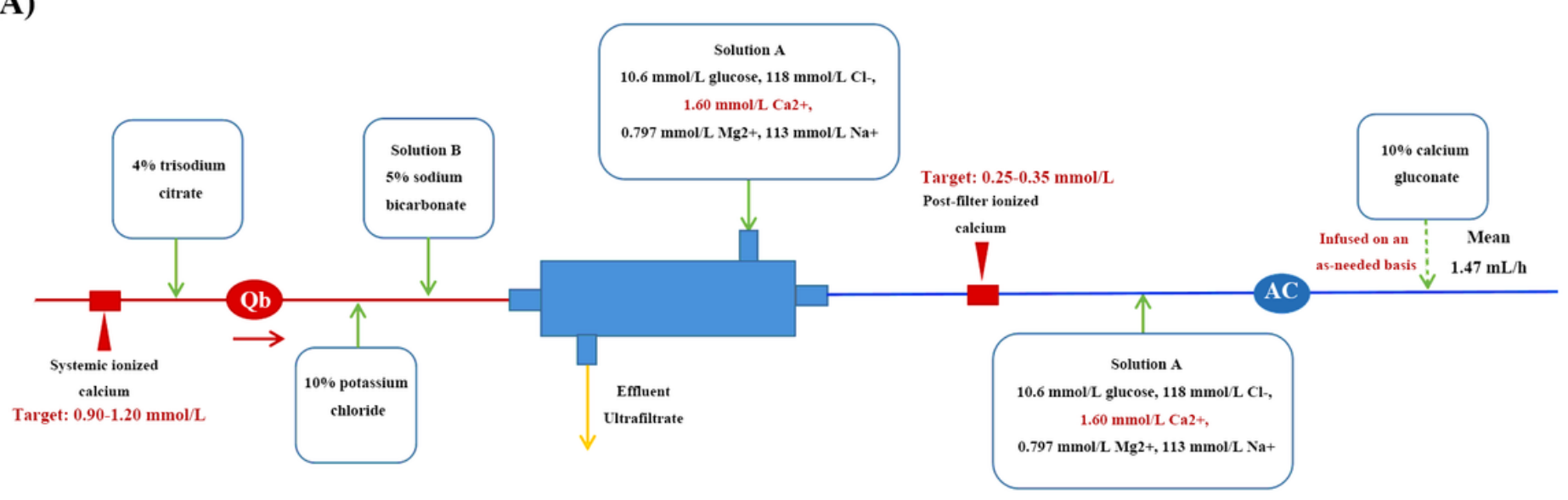

(B)

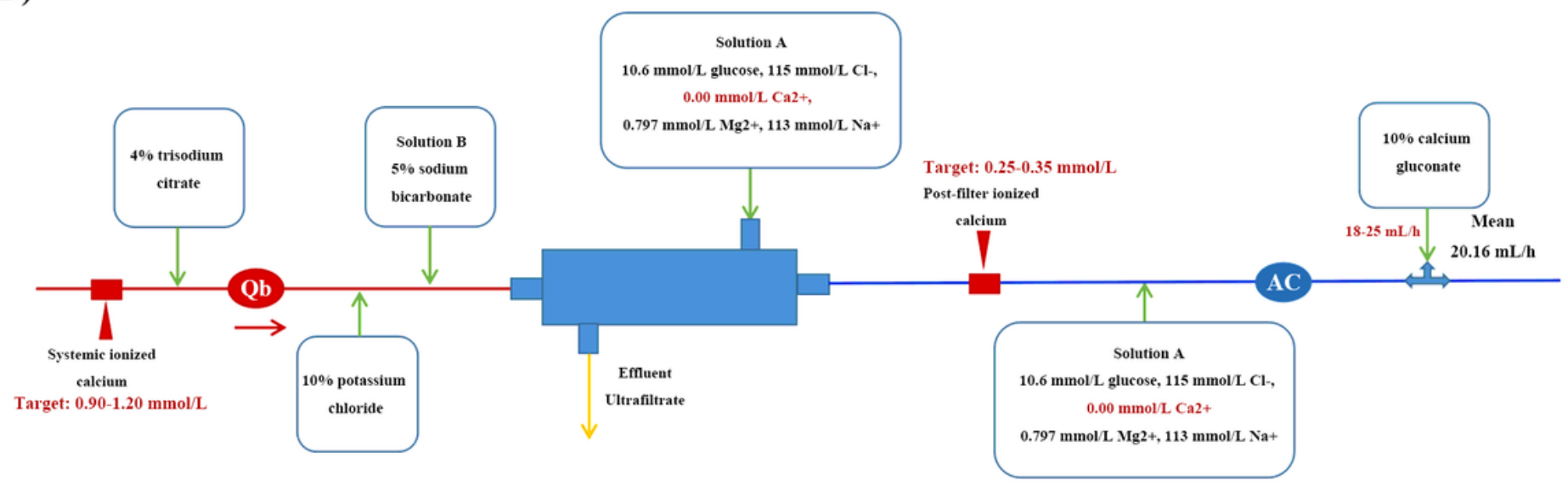

\section{Figure 1}

The standard scheme for application of calcium-containing (a) and calcium-free replacement solutions (b) in RCA based post-dilution CVVHDF. $4 \%$ trisodium citrate solution, $10 \%$ potassium chloride and solution B ( $5 \%$ sodium bicarbonate) were infused via the pre-blood pumps. Calcium-containing and calcium-free replacement solutions were used as both replacement solutions and dialysates (delivered post-filter). $10 \%$ calcium gluconate was infused on an as-needed basis as an intermittent intravenous bolus in calcium-containing group (a). In calcium-free replacement group, intravenous $10 \%$ calcium gluconate was administered continuously by a pump via a T-branch pipe in post-filter (b). RCA, regional citrate anticoagulation; CVVHDF, continuous venovenous hemodiafiltration; Qb, blood flow rate; AC, airtrap chamber. 


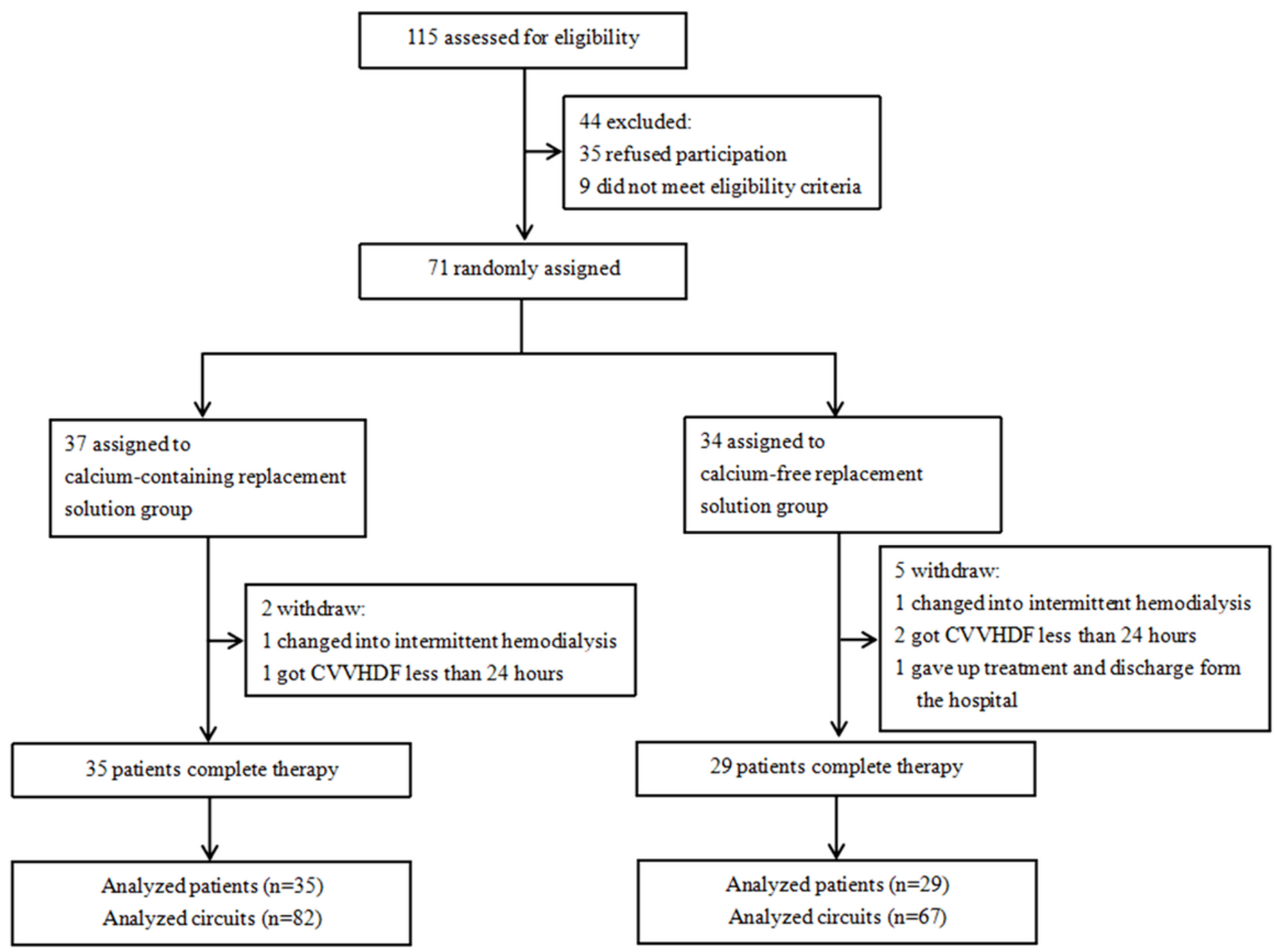

Figure 2

Flow diagram of a participant's involvement. 
a

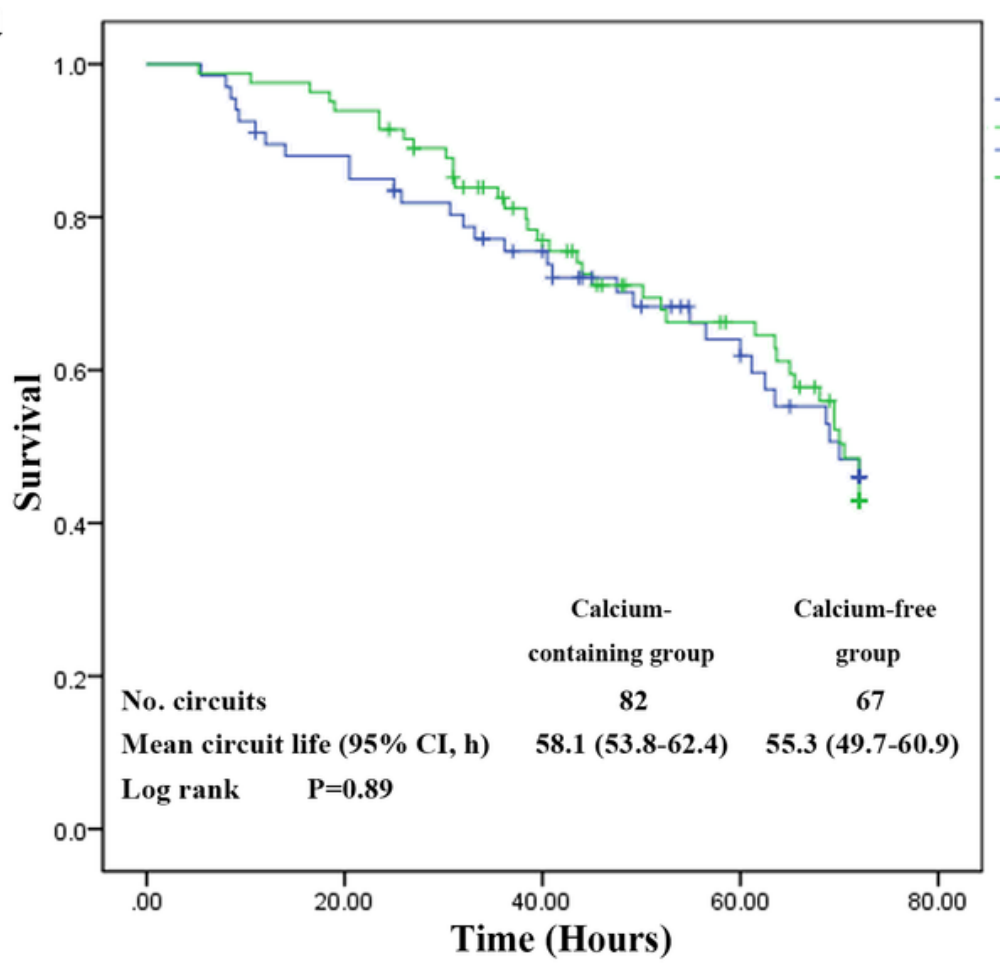

$\neg$ Calcium-free group

$\neg$ Calcium-containing group

+ Calcium-free group-censored

+ Calcium-containing group-censored

b

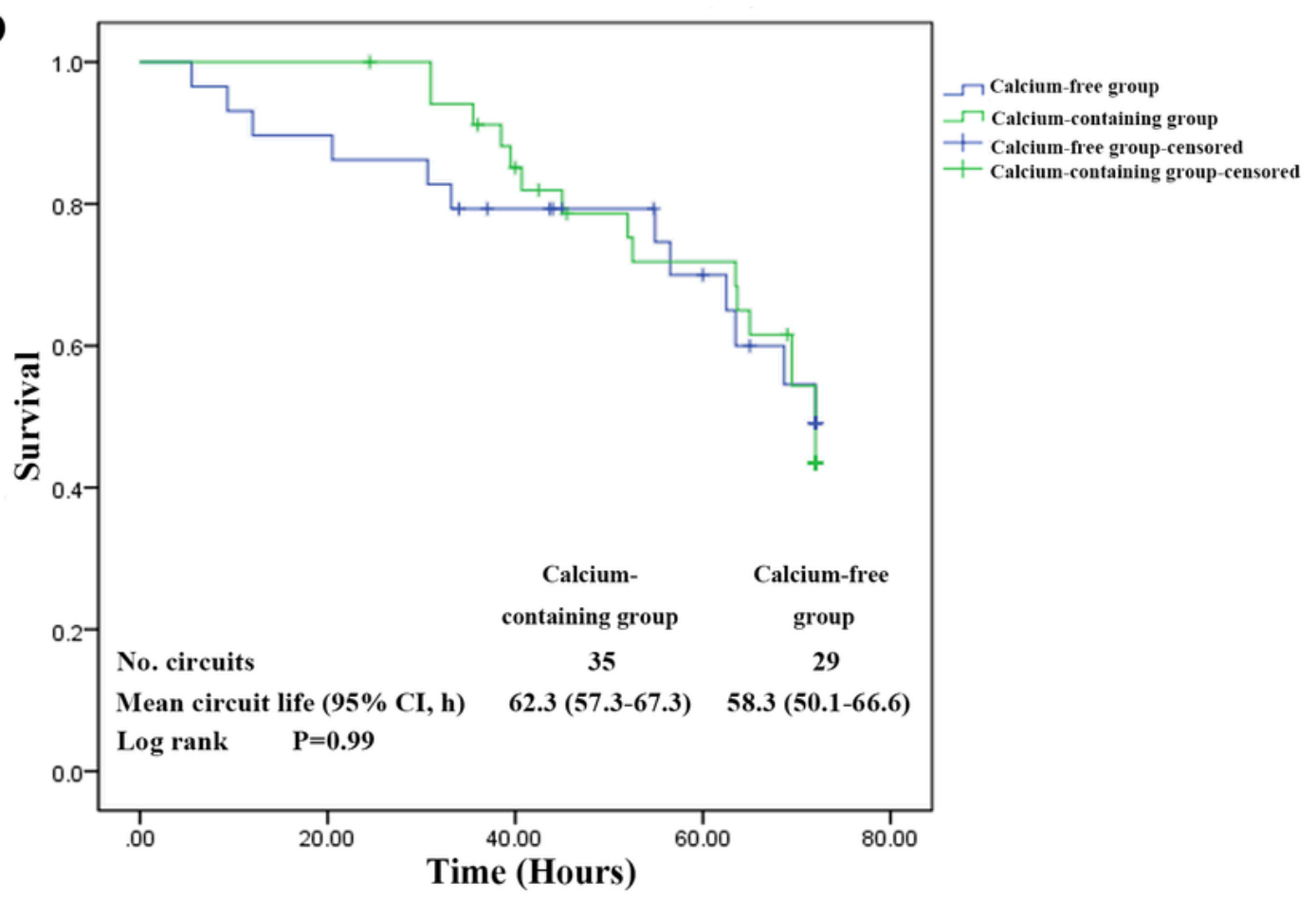

\section{Figure 3}

The Kaplan-Meier curve for all of the hemofilter life span (a) and the first circuit life span (b).

\section{Supplementary Files}

This is a list of supplementary files associated with this preprint. Click to download. 
- 0825SupplementaryFig1.tif

Page 25/25 